\title{
Excess iron undermined bone load-bearing capacity through tumor necrosis factor- $\alpha$-dependent osteoclastic activation in mice
}

\author{
JUNPING LI ${ }^{1,2^{*}}$, YANLI HOU ${ }^{1,2^{*}}$, SHUPING ZHANG ${ }^{2}$, HONG JI $^{1}$, HAIQIN RONG ${ }^{1}$, \\ GUANGBO QU ${ }^{2}$ and SIJIN LIU ${ }^{2}$ \\ ${ }^{1}$ Shandong Institute of Endocrine and Metabolic Diseases, Jinan 250062; ${ }^{2}$ State Key Laboratory \\ of Environmental Chemistry and Ecotoxicology, Research Center for Eco-Environmental Sciences, \\ Chinese Academy of Sciences, Beijing 100085, P.R. China
}

Received June 25, 2012; Accepted July 31, 2012

DOI: $10.3892 /$ br. 2012.6

\begin{abstract}
Iron overload has been associated with bone mass loss. To elucidate the effects of excess iron on bone metabolism, an iron-overloading mouse model was established by administering iron-dextran at $250 \mathrm{mg} / \mathrm{kg}$ to female BALB/c mice. After 4 weeks, the mice were sacrificed and the biomechanical properties of the femurs were examined. The results suggested a notable decrease of the maximal bending stress and the modulus of bending elasticity in the femurs obtained from the excess iron-treated mice compared to the control mice. The levels of the serum osteocalcin, C-telopeptide of type I collagen (CTX-1) and tumor necrosis factor- $\alpha$ (TNF- $\alpha$ ) were measured in order to investigate the underlying mechanism responsible for the excess iron-induced bone strength reduction. Overall, the results suggested that iron overload resulted in a marked reduction of bone load-bearing capacity through a TNF-triggered osteoclast differentiation and resorption mechanism.
\end{abstract}

\section{Introduction}

Bone mass is maintained by the balance of bone formation and resorption, involving a number of regulatory pathways. Bone formation is performed by osteoblasts, while bone resorption is carried out by osteoclasts. Disruptions of these processes are likely to induce hyperosteogeny or osteopenia $(1,2)$. Numerous

Correspondence to: Professor Guangbo Qu, State Key Laboratory of Environmental Chemistry and Ecotoxicology, Research Center for Eco-Environmental Sciences, Chinese Academy of Sciences, 18 Shuangqing Road, Haidian, Beijing 100085, P.R. China

E-mail: qbqu@rcees.ac.cn

Dr Haiqin Rong, Shandong Institute of Endocrine and Metabolic Diseases, Jinan 250062, P.R. China

E-mail: haiqinrong@126.com

*Contributed equally

Key words: iron overload, bone mass, bone resorption, bone strength clinical studies reported that patients with osteoporosis showed iron accumulation in the bone, potentially leading to pathological damages, such as occurred femoral neck fractures in patients of African descent (3-5).

Ovariectomized rats with an increased iron level showed a higher tendency to have osteoporosis (6). Tsay et al (7) reported that the iron overload (IO)-induced bone loss in mice was correlated with the inflammatory bone resorption and oxidative stress. Using in vitro cell assays, iron treatment was demonstrated to inhibit osteoblast formation, proliferation and mineralization (8-10), while promoting osteoclast differentiation and increased osteoclastic function (11). Therefore, IO is believed to disrupt bone metabolism and induce bone loss.

Iron accumulation in tissue is likely to provoke the formation of reactive oxygen species (ROS) (12), while the iron excess is likely to induce an increased expression of tumor necrosis factor- $\alpha$ (TNF- $\alpha$ ) (13). However, the contribution of oxidative stress to osteoporosis was also recognized $(14,15)$. ROS induced increased TNF- $\alpha$ expression and activated several signaling systems involved in the osteoclastic differentiation, especially the nuclear factor- $\kappa \mathrm{B}(\mathrm{NF}-\kappa \mathrm{B})$ pathway. TNF- $\alpha$ was found to synergize strongly with the receptor activator of $\mathrm{NF}-\kappa \mathrm{B}$ ligand in osteoclast formation and activation $(16,17)$. Therefore, osteoclastic hyperresorption was highly involved in ROS- and TNF- $\alpha$-induced bone loss. However, whether IO is linked to induced bone function impairment has yet to be investigated.

In this study, a mouse model with IO was developed and was used to determine whether the excess iron undermined bone strength and load-bearing capacity through TNF- $\alpha$ induction and osteoclastic function promotion.

\section{Materials and methods}

Animal experiments. Six-week-old female BALB/c mice were fed under sterile conditions and divided into two groups. The iron excess (i.e., IO), group was injected with iron-dextran (Sigma, St. Louis, MO, USA) at $250 \mathrm{mg} / \mathrm{kg}$. The control mice were injected with equal amounts of phosphate-buffered saline (PBS) twice a week for four weeks. Subsequent to sacrificing the animals at $48 \mathrm{~h}$ after the final injection, blood and femur samples were collected for analysis. 
A

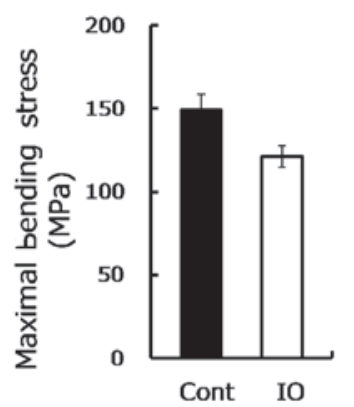

B

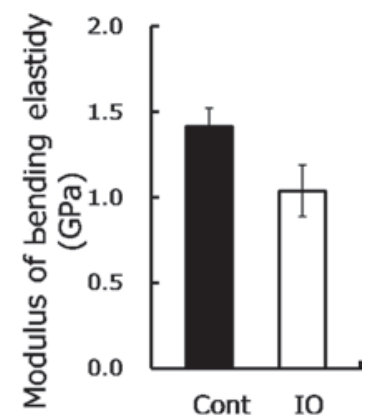

Figure 1. Excess iron impaired biomechanical properties of femurs from mice with IO are shown. (A) Maximal bending stress was measured by the three-point bending test for femurs from mice with IO treatment and control mice (n=8-9). (B) Modulus of bending elasticity using the three-point bending test for femurs upon IO condition $(n=8-9)$ is shown.

Assessments of bone metabolic markers. Blood samples were collected from the heart and allowed to clot at room temperature. Serum samples were separated from the blood by centrifugation at 3,000 rpm for $10 \mathrm{~min}$. Serum osteocalcin, C-telopeptide of type I collagen (CTX-1) and TNF- $\alpha$ were measured by ELISA (Rapidbio; RapidBio, West Hills, CA, USA; R\&D Systems, Minneapolis, MN, USA), according to the manufacturer's instructions.

Bone biomechanical analysis. Biomechanical properties of femurs were measured by the three-point bending test performed using a universal material test machine (AG-1S; Shimadzu Co., Kyoto, Japan) at room temperature, as described in previous studies (18-20). Briefly, the diaphysis was placed on two supports with a span of $10 \mathrm{~mm}$, while the loading pin was centered above two supports at a displacement rate of $2 \mathrm{~mm} / \mathrm{min}$, until fracture occurred.

Statistical analysis. The two-tailed Student's t-test was used to analyze experimental data. The data were presented as the means \pm standard error (SE). $\mathrm{P}<0.05$ was considered to indicate a statistically significant difference.

\section{Results and Discussion}

Mechanical loading sends signals which are essential for bone mass maintenance and strength depending on the stimulus (21). To determine whether IO undermines the bone strength and function, the biomechanical parameters of the bone were measured. Maximal bending stress is the maximum load per unit of bone area in the plastic deformation stage, while the modulus of bending elasticity involves the resistance to
A

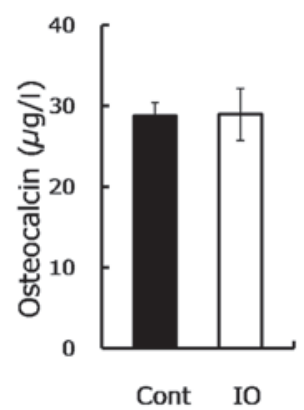

B

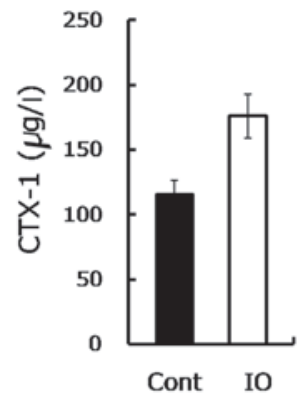

Figure 2. Excess iron provoked bone resorption. (A) Serum osteocalcin assayed by ELISA in the groups $(n=9)$ is shown. (B) CTX-1 assessed by ELISA in the two groups $(n=5-9)$ is shown.

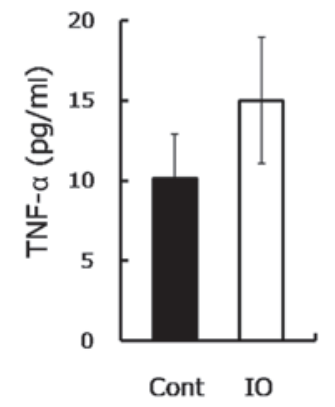

Figure 3. Excess iron increased serum TNF- $\alpha$. Serum TNF- $\alpha$ measured by ELISA from mice with IO treatment and the control mice $(n=8)$ is shown.

elastic deformation of the bone, reflecting the inner strength of the bone, without size effect. As shown in Fig. 1A, the maximal bending stress was reduced approximately by $20 \%$ $(\mathrm{P}=0.03)$ under IO conditions, while the modulus of bending elasticity was reduced by $30 \%$ ( $P=0.05$, Fig. 1B). These findings suggested that IO led to a significant reduction of bone load-bearing capacity and thus increased the risk of fractures.

Bone load-bearing capacity is dependent on bone formation, as well as bone resorption. To gain insight into the mechanism underlying the IO-mediated impairment to bone load-bearing capacity, the osteocalcin and CTX-1 were measured. Osteocalcin is secreted by osteoblasts in the maturation stage to sustain mineralization and may be used as a marker of bone formation in the evaluation of bone mass. The findings of this study showed no statistically significant difference in the osteocalcin content between the mice with IO and the control group (28.82 vs. $28.99, \mathrm{P}=0.963$, Fig. 2A). In contrast to these findings, in their study, Yamasaki et al (8) 


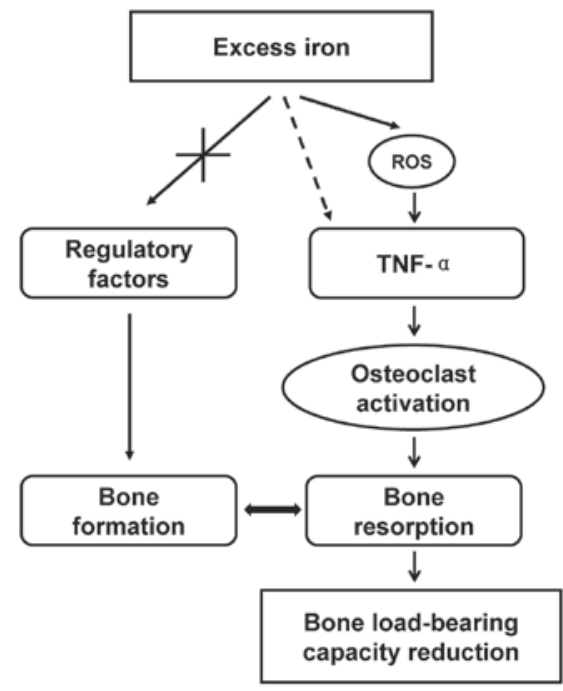

Figure 4. A schematic delineation of the mechanism responsible for IO-triggered impairment to bone strength is shown. IO largely increased serum TNF- $\alpha$ content by stimulating inflammatory cells through ROS induction. Consequently, increased TNF- $\alpha$ promoted osteoclastogenesis, then activated osteoclastic function stimulated bone resorption. However, excess iron had little effect on bone formation. As a result, bone mass was reduced and bone load-bearing capacity was undermined.

demonstrated that excess iron reduced the mineralization of rat calvarial osteoblast-like cells by showing a decreased number of mineralized nodules $(9,10)$. This discrepancy may be due to the in vitro sensitivity of osteoblasts being higher compared to the in vivo, when directly exposed to iron. Thus, consistent with another in vivo study (7), our results demonstrated that IO had little effect on bone formation.

CTX-1 is the degraded product of type I collagen with an enhanced osteoclastic activity, used to evaluate the bone resorptive process. In the present study, the CTX-1 content was observed to be notably increased $(\sim 50 \%)$ in the IO group compared to the control group $(\mathrm{P}=0.008$, Fig. $2 \mathrm{~B})$, suggesting an elevated bone resorption.

The increased bone resorption indicated that the osteoclastic activity was stimulated. Since IO was demonstrated to induce the TNF- $\alpha$ expression that further triggers osteoclastic function $(13,22,23)$, the TNF- $\alpha$ level was measured. Consistent with a previous study (13), the TNF- $\alpha$ level in the IO mice was approximately 1.5 -fold higher compared to the control group (Fig. 3), as shown. TNF- $\alpha$ was demonstrated to be involved in the enhancement of the development of osteopenia and osteoporosis $(14,15)$. TNF- $\alpha$ promoted osteoclastogenesis through synergizing with a permissive level of the receptor activator of $\mathrm{NF}-\kappa \mathrm{B}$ ligand (RANKL), as well as through directly affecting osteoclasts $(16,17)$. In the current study, a high serum TNF- $\alpha$ level was observed in mice with IO. The increased TNF- $\alpha$ is likely to promote the osteoclastic function and enhance inflammatory osteolysis, resulting in advanced bone resorption (24). Therefore, upon IO exposure, although increased TNF- $\alpha$ was not involved in mineralization inhibition, it accelerated bone loss and eventually reduced bone strength.

In conclusion, the findings of the present study have shown that IO undermined bone load-bearing capacity through the enhancement of TNF- $\alpha$ secretion, which facilitated osteoclastic differentiation and promoted the bone-resorbing activity (Fig. 4). Therefore, decreasing the iron burden is likely to be a promising approach for the treatment of bone metabolic diseases, such as osteopenia and osteoporosis.

\section{Acknowledgements}

This study was financed by grants from the Chinese Academy of Sciences (KZCX2-EW-404) and the National Natural Science Foundation of China (nos. 21077128, 20921063 and 21177151). The authors would like to thank the laboratory staff members for their assistance with the experiments and reagents.

\section{References}

1. Mountzios G, Dimopoulos MA, Bamias A, Papadopoulos G, Kastritis E, Syrigos K, Pavlakis G and Terpos E: Abnormal bone remodeling process is due to an imbalance in the receptor activator of nuclear factor-kappa B ligand (RANKL)/osteoprotegerin (OPG) axis in patients with solid tumors metastatic to the skeleton. Acta Oncol 46: 221-229, 2007.

2. Fohr B, Dunstan CR and Seibel MJ: Clinical review 165: markers of bone remodeling in metastatic bone disease. J Clin Endocrinol Metab 88: 5059-5075, 2003.

3. Maurer J, Harris MM, Stanford VA, Lohman TG, Cussler E, Going SB and Houtkooper LB: Dietary iron positively influences bone mineral density in postmenopausal women on hormone replacement therapy. J Nutr 135: 863-869, 2005.

4. Schnitzler CM, Schnaid E, MacPhail AP, Mesquita JM and Robson HJ: Ascorbic acid deficiency, iron overload and alcohol abuse underlie the severe osteoporosis in black African patients with hip fractures - a bone histomorphometric study. Calcified Tissue Int 76: 79-89, 2005.

5. Valenti L, Varenna M, Fracanzani A, Rossi V, Fargion S and Sinigaglia L: Association between iron overload and osteoporosis in patients with hereditary hemochromatosis. Osteoporosis Int 20: 549-555, 2009.

6. Liu G, Men P, Kenner GH and Miller SC: Age-associated iron accumulation in bone: implications for postmenopausal osteoporosis and a new target for prevention and treatment by chelation. Biometals 19: 245-251, 2006.

7. Tsay J, Yang Z, Ross FP, Cunningham-Rundles S, Lin H, Coleman R, Mayer-Kuckuk P, Doty SB, Grady RW, Giardina PJ, Boskey AL and Vogiatzi MG: Bone loss caused by iron overload in a murine model: importance of oxidative stress. Blood 116: 2582-2589, 2010.

8. Yamasaki $\mathrm{K}$ and Hagiwara $\mathrm{H}$ : Excess iron inhibits osteoblast metabolism. Toxicol Lett 191: 211-215, 2009.

9. Messer JG, Kilbarger AK, Erikson KM and Kipp DE: Iron overload alters iron-regulatory genes and proteins, down-regulates osteoblastic phenotype, and is associated with apoptosis in fetal rat calvaria cultures. Bone 45: 972-979, 2009.

10. Yang Q, Jian J, Abramson SB and Huang X: Inhibitory effects of iron on bone morphogenetic protein 2-induced osteoblastogenesis. J Bone Miner Res 26: 1188-1196, 2011.

11. Ishii KA, Fumoto T, Iwai K, Takeshita S, Ito M, Shimohata N, Aburatani H, Taketani S, Lelliott CJ, Vidal-Puig A and Ikeda K: Coordination of PGC-1beta and iron uptake in mitochondrial biogenesis and osteoclast activation. Nat Med 15: 259-266, 2009.

12. Galaris D and Pantopoulos K: Oxidative stress and iron homeostasis: mechanistic and health aspects. Crit Rev Clin Lab Sci 45: $1-23,2008$.

13. Andrews $M$ and Arredondo M: Hepatic and adipocyte cells respond differentially to iron overload, hypoxic and inflammatory challenge. Biometals 25: 749-759, 2012.

14. Lean JM, Davies JT, Fuller K, Jagger CJ, Kirstein B, Partington GA, Urry ZL and Chambers TJ: A crucial role for thiol antioxidants in estrogen-deficiency bone loss. J Clin Invest 112: 915-923, 2003.

15. Jagger CJ, Lean JM, Davies JT and Chambers TJ: Tumor necrosis factor-alpha mediates osteopenia caused by depletion of antioxidants. Endocrinology 146: 113-118, 2005.

16. Fuller K, Murphy C, Kirstein B, Fox SW and Chambers TJ: TNFalpha potently activates osteoclasts, through a direct action independent of and strongly synergistic with RANKL. Endocrinology 143: 1108-1118, 2002. 
17. Lam J, Takeshita S, Barker JE, Kanagawa O, Ross FP and Teitelbaum SL: TNF-alpha induces osteoclastogenesis by direct stimulation of macrophages exposed to permissive levels of RANK ligand. J Clin Invest 106: 1481-1488, 2000.

18. Mattila P, Knuuttila M, Kovanen V and Svanberg M: Improved bone biomechanical properties in rats after oral xylitol administration. Calcif Tissue Int 64: 340-344, 1999.

19. Zhang L, Liu Y, Wang D, Zhao X, Qiu Z, Ji H and Rong H: Bone biomechanical and histomorphometrical investment in type 2 diabetic Goto-Kakizaki rats. Acta Diabetol 46: 119-126, 2009.

20. Niu YB, Li YH, Kong XH, Zhang R, Sun Y, Li Q, Li C, Liu L, Wang J and Mei QB: The beneficial effect of Radix Dipsaci total saponins on bone metabolism in vitro and in vivo and the possible mechanisms of action. Osteoporos Int: Apr 26, 2012 (Epub ahead of print). doi: 10.1007/s00198-012-1932-y.
21. Rubin C, Turner AS, Mallinckrodt C, Jerome C, McLeod K and Bain S: Mechanical strain, induced noninvasively in the high-frequency domain, is anabolic to cancellous bone, but not cortical bone. Bone 30: 445-452, 2002.

22. Blair HC and Zaidi M: Osteoclastic differentiation and function regulated by old and new pathways. Rev Endocr Metab Disord 7: 23-32, 2006.

23. Zhao B, Grimes SN, Li S, Hu X and Ivashkiv LB: TNF-induced osteoclastogenesis and inflammatory bone resorption are inhibited by transcription factor RBP-J. J Exp Med 209: 319-334, 2012.

24. Vattakuzhi Y, Abraham SM, Freidin A, Clark AR and Horwood NJ: Dual specificity phosphatase 1 null mice exhibit spontaneous osteolytic disease and enhanced inflammatory osteolysis in experimental arthritis. Arthritis Rheum 64: 2201-2210, 2012. 